Research Article

\title{
High-Performance Computational Early Warning Analysis of Agricultural Economy Relying on Binary Fuzzy Cluster Analysis Algorithm
}

\author{
Fang Tang 10 \\ School of Business Administration, Xinyang Agriculture and Forestry University, Xinyang 464000, China \\ Correspondence should be addressed to Fang Tang; 2001250022@xyafu.edu.cn
}

Received 15 October 2021; Revised 2 November 2021; Accepted 5 November 2021; Published 23 November 2021

Academic Editor: Miaochao Chen

Copyright (C) 2021 Fang Tang. This is an open access article distributed under the Creative Commons Attribution License, which permits unrestricted use, distribution, and reproduction in any medium, provided the original work is properly cited.

\begin{abstract}
In this paper, a binary fuzzy cluster analysis algorithm is used for an in-depth study and analysis of high-performance computational early warning in the agricultural economy. The definition of interval type-two fuzzy set and its operation are summarized. Considering the uncertain information that will be encountered in the evaluation process, this paper constructs the evaluation model of rural informatization construction performance based on interval type-two fuzzy numbers, which improves the accuracy of the evaluation results. The fuzzy clustering centres are modified according to the nature of the fuzzy clustering centre matrix, and the optimal fuzzy clustering centres and optimal fuzzy clustering division matrix with consistent order are solved. Using the level eigenvalues to find out in the abundant water period, there are 4 monitoring sections of water quality evaluation results for still clean and 5 monitoring sections of water quality evaluation results for slight pollution. In the flat-water period, there are 2 monitoring sections of the water quality evaluation results that are still clean and 7 monitoring sections of the water quality evaluation results are slightly polluted. In the dry water period, the water quality evaluation results of the 9 monitoring sections are slightly polluted. The results and the use of integrated pollutant index evaluation method results are consistent, indicating that the use of fuzzy clustering model for water quality evaluation is practical and effective. Its functional structure includes management business, innovation business, service business, production business, and operation business functions, in addition to the design of the collection and storage scheme based on Hadoop data warehouse, to achieve accurate matching of information of science and technology agricultural services in the whole agricultural industry chain. Finally, the implementation and maintenance of the cloud-based science and technology agricultural service information system are deployed, and the operational effects of its information system-related functions are demonstrated using prototype design. This paper constructs a comprehensive information system for science and technology agricultural services based on cloud technology that integrates management, innovation, service, production, and operation, which meets not only the needs of traditional science and technology agricultural service information systems, such as expert consultation and training demonstration, but also other needs spawned by the development of agricultural modernization.
\end{abstract}

\section{Introduction}

Carrying out services for the benefit of farmers in science and technology is of great significance to improving the contribution of scientific and technological progress, helping to modernize agriculture, and achieving the strategic goal of building a moderately prosperous society in all aspects. With the development of information technology, it has become common to carry out services for the benefit of agriculture with the help of information technology for agricultural services [1]. The Informa ionization of science and technology services for agriculture is an activity carried out in the process of promoting agricultural science and technology informatization, which integrates the use of various information technology means to collect, analyse, and provide relevant information services for science and technology information resources for agriculture. It includes collecting, analysing, and providing related information services of 
scientific and technological benefiting agricultural information resources. The new driving force for promoting the informatization of scientific and technological benefiting agricultural services is agricultural technological innovation. Agricultural technological innovation aims to increase land output rate, resource utilization rate, and labour productivity. The new dynamic energy to promote the Informa ionization of science and technology services for agriculture is agricultural science and technology innovation, which aims to improve land output rate, resource utilization rate, and labour productivity, with breakthrough points in R\&D targeting the frontier science and technology needs of agriculture and transforming agricultural science and technology achievements into practices to solve agricultural production problems. Informa ionization of scientific and technological agricultural services is conducive to promoting innovation and transformation of agricultural science and technology, guiding agricultural production personnel to use agricultural science and technology to improve the efficiency and quality of agricultural production, providing a large number of information resources on scientific and technological agricultural services for decision-making by agricultural-related administrative departments, further promoting the strength of demand-oriented technological innovation, facilitating the transformation of science and technology into productivity, and all-round enhancing the development level of agricultural science and technology and promoting the agricultural modernization process [2]. However, there are still some problems in the operation of the information system of science and technology services for agriculture, from the production scenario, excluding the irresistible factors, the agricultural production site information cannot be fed back in real time, waiting for the personnel of agriculture-related service units to provide diagnostic services at home, which has missed the best time to prevent and control disasters and reduced crop productivity. In the process of agricultural production, the use of chemical fertilizers and pesticides lacks records, and excessive use causes waste of resources and environmental pollution [3]. From the perspective of construction units, science and technology service systems for the agricultural benefit independently developed by various construction units and isolated from each other, resulting in low utilization of information systems for science and technology service for agricultural benefit and waste of IT resources. From the technical point of view, various information systems of science and technology services for agriculture use different architectures, programming languages, and storage formats, which are not conducive to data exchange between systems, and it is difficult to share and reuse information resources of science and technology services for agriculture.

The degree of informatization is one of the important criteria for measuring the degree of economic development, cultural heritage, and modernization of a country or a region. Informa ionization has gradually become the main driving force of national economic growth and social development and is an important strategic resource in the competition of each country or region [4]. The construction of rural informatization is in line with the policy idea of building new socialist countryside and modernizing agriculture, which is to implement the strategy of revitalizing the countryside, focusing on the simultaneous development of new industrialization, informatization, urbanization, and agricultural modernization; promote the comprehensive upgrading of agriculture, the comprehensive progress of rural areas, and the comprehensive development of farmers; and accelerate the modernization of agriculture and rural areas. Moreover, the construction of rural informatization is an important means to realize the deployment of a rural revitalization strategy. At the same time, rural informatization has been written into the Central Government's Document No. 1 many times, which points out that the construction of rural informatization should not just be comprehensively promoted, relying on information technology to infer innovation. Closely integrating information flow with technology flow, capital flow, talent flow, and material flow and promoting the optimization of resource allocation will certainly play an active role in promoting the development of science and technology, innovating the form of economic development, and adjusting the supply-side structure so that more fruits of economic development can benefit the people. By building a fast track for information services through the integration of three networks, grassroots information service stations, and demonstration villages for rural informatization, the working environment for the construction of rural informatization is constantly optimized.

The existing research work on the evaluation of rural informatization performance lacks scientific basis, comprehensiveness, operability, and comparability and has more obvious defects. In recent years, several scholars have conducted theoretical and practical exploration of agricultural and rural informatization construction work and formed a series of models, which provide the theoretical reference for our current study. However, when examined in terms of the scientific and comprehensive nature of the operation, the existing models still have certain shortcomings. They do not match the current situation of rural informatization construction in the context of the new era. This study understands the basic situation of rural informatization construction from the perspective of two main rural informatization service subjects: farmers and rural enterprises. Because these two subjects lack professional knowledge and have no access to specific data on informatization construction, they tend to use linguistic values, such as "very good, good," to evaluate the performance indicators. In this paper, by introducing interval type-II fuzzy numbers, the linguistic values used by evaluators for performance indicators transformed into interval type-II fuzzy numbers, and the uncertainty information in the actual situation is scientifically quantified. The evaluation model is constructed by combining the relevant decisionmaking methods of interval type-two fuzzy numbers to provide an efficient evaluation model and new ideas for evaluating rural information construction performance. Constructing a reasonable performance evaluation index system for rural informatization construction work is used 
to measure the performance of rural informatization work and achieve the development of scientific and effective rural informatization development policies through performance evaluation, which is the requirement of the party and government for rural informatization construction today. In constructing the evaluation index system, this study conducted expert group meetings by inviting experts from different fields to form an expert group to make suggestions on the construction of performance evaluation indexes for rural informatization construction. Combined with the experts' suggestions, this study uses a mixed index system construction method to determine the evaluation.

\section{Status of Research}

The soil water evaporation can maintain the humidity of the air near the ground and reduce the air temperature. Plants use soil water to meet their growth requirements. In contrast, plants can convert carbon dioxide and water into oxygen and organic matter after photosynthesis, releasing a large amount of oxygen while providing energy for phytophagous animals and maintaining the balance of nature [5]. Therefore, it will be of great strategic importance to the sustainable development of agriculture to reasonably develop and utilize soil water resources, to explore the potential of soil water resources development and its ability to support agricultural production and maintain a good agricultural ecological environment while giving full play to the maximum advantages of soil water resources [6]. In the above concept, soil water is considered a recoverable freshwater resource, and soil water resources have the characteristics of natural resources and the dynamic properties of water balance. Meanwhile, the amount of soil water resources can be increased by implementing good hydraulic soil modification measures and agricultural technology measures to increase rainfall infiltration on farmland and reduce surface runoff. However, Tripathi and Maktedar believed that soil water resources are the difference between precipitation and surface runoff and that groundwater resources are part of soil water resources. Most scholars have not accepted this view. Further research has been conducted on soil water resources, and significant progress has been made. In addition to recognizing soil water as a natural resource, it was proposed that "soil water is the most important natural resource" from the viewpoint of its role in the interchange of land water and environmental factors and agricultural production, which was recognized by scholars at home and abroad [7]. While the production of mariculture is increasing, the environmental problems caused by it are gradually coming to the fore, mainly in two aspects. Firstly, the excessive use of various nutrients in the farming process has led to changes in the water quality parameters of the farmed sea, thus increasing the eutrophication of seawater. Secondly, the scope of the farming area is not planned rationally, resulting in the reduction of the area of near-shore natural wetlands, and the remaining farming bait in the bottom mud will ferment and produce harmful substances, thus hindering the flourishing of various aquatic organisms [8].
The theory of type two fuzzy sets is continuously updated and refined to define a similarity measure for type two fuzzy sets, and the method is used to assess the similarity of student work [9]. A hierarchical analysis method in this fuzzy environment is proposed by combining type two fuzzy sets with linguistic variables. A comparative analysis with type one fuzzy sets is conducted to verify the superiority of type two fuzzy sets. The set of type two fuzzy sets, the rules of algebraic operations, and the properties of the affiliation function are discussed [10]. The representation of type-two fuzzy numbers is newly defined in reference [11], by which theorems for the intersection, merge, and complement operations of type-two fuzzy numbers are derived without using the extension principle [11]. The intersection, merge, and complement operations for interval type-two fuzzy sets are defined, while the footprint uncertainty is proposed, and it is pointed out that the footprint uncertainty is the plane region constituted by the set of principal affiliation degrees [12]. Several methods for fuzzy measurement of interval type-two fuzzy numbers are investigated, in which the centralized approach combines the KM algorithm to reduce the time complexity of computation by dimensionality reduction. Several different ways of measuring interval typetwo fuzzy numbers are proposed, respectively. In contrast, problems in previous studies are pointed out, and new methods are proposed to compensate for the shortcomings of the old ones [13].

Throughout the above studies, scholars at home and abroad mainly interpret the meaning of rural informatization from the concept, characteristics, and research significance of rural informatization, by establishing the index system, data collection, and data analysis and establishing a model to evaluate the performance of rural informatization construction, including the comprehensive index method, influence factor analysis, factor analysis, and other methods. However, when examined in terms of the scientific and comprehensiveness of the operation, the existing evaluation system still has certain shortcomings and does not match the current situation of rural informatization construction in the context of the new era. In addition, in the actual situation, the process of evaluating the performance of rural informatization construction often encounters much uncertain information, and there are few studies in the existing literature on how to deal with such uncertain information.

\section{Binary Fuzzy Cluster Analysis Algorithm for High-Performance Computing Early Warning Design for Agricultural Economy}

\subsection{Binary Fuzzy Clustering Analysis Algorithm Construction.} According to the findings of systems science, the integrity of each system consists of the elements, structural classifications, and recursive relationships that exist in the system. There is a close connection between each level in the system, and when one level changes, the other levels react accordingly. Sometimes, the role between multiple levels requires intermediary levels to coordinate. Thus, the role of intermediary levels is very important in systems science [14]. 
Reducing the productivity of crops: in the process of agricultural production, the use of chemical fertilizers and pesticides is lacking in records, and excessive use causes waste of resources and environmental pollution. The fuzzy clustering iterative model is based on the concept of weighted generalized Euclidean power distance, the objective function is established between the whole data sample and the classification level, the initial superiority matrix is given according to the property requirements in the fuzzy clustering iterative model, and the computational accuracy that meets the iteration requirements is given. The optimal fuzzy clustering matrix and the optimal fuzzy clustering centre that meet the accuracy requirements can be solved by multiple iterations, and the fuzzy clustering centres are adjusted for consistency and evaluated with level eigenvalues. The optimal fuzzy clustering matrix and the optimal fuzzy clustering centre meet the accuracy requirement. The fuzzy clustering centre is adjusted for consistency and evaluated with the level eigenvalues:

$$
R=\frac{x_{\max }-x_{\min }}{x_{\max }+x_{\min }}
$$

According to the connotation of soil water resources carrying capacity of arable land, the target of soil water resources carrying is the capacity of arable land in agricultural production. For this reason, the economic benefit target mainly considers agricultural economic output value. In contrast, the social benefit target mainly considers food security issues and agricultural water conservation, and two indicators of total food production and agricultural water use ratio are used. Therefore, this paper takes agricultural economic output value, total food production and agricultural water use ratio as the objectives, crop planting area as the decision variables, and crop sowing area and total agricultural irrigation water use as the constraints and establishes a multiobjective optimization model of soil water resources carrying capacity of arable land:

$$
\begin{aligned}
F_{1} & =\lim _{m \longrightarrow \infty} \sum_{i, j=1}^{m}\left(x_{i} y_{i} p_{i}-x_{j} y_{j} p_{j}\right), \\
\min F & =\operatorname{Sup}\left\{\lim _{n \longrightarrow \infty} \frac{1}{W} \sum_{i=1}^{n} \frac{x_{i} y_{j} p_{j}}{\eta_{j}}\right\} .
\end{aligned}
$$

The particle swarm algorithm models the solutions of the problem all as particles in the search space, which can ignore their volume and mass and fly at a determined speed. The particles choose a suitable computational function to find their fitness and compare it with the fitness value of the swarm's current optimal point, dynamically adjust their flight speed and corresponding point location, and finally search for the global optimal point. It will certainly play a positive role in promoting technological development, innovating economic development forms, and adjusting the supply-side structure so that more economic development results will benefit the people. The type-two fuzzy number is an extension of the traditional type-one fuzzy set, which can portray a higher degree of fuzziness more delicately. While interval type-II fuzzy numbers are a special case of type-II fuzzy numbers, the values of their subordinate degrees are both 1. Type-II fuzzy numbers are jointly inscribed by primary and secondary subordinate degrees. This section briefly reviews the concepts related to type two fuzzy numbers, interval type-two fuzzy numbers, as shown in Figure 1.

The basic principle of the performance evaluation index system is that it should objectively and comprehensively reflect the whole process of rural informatization construction and the laws of rural informatization construction, revealing its essential characteristics [15]. The construction of the performance evaluation index system must comprehensively consider that the evaluation system should include all the key components that affect the construction of rural informatization and strictly develop the process of activities to ensure that the performance evaluation index system is fair and effective and scientific and reasonable. At the same time, the results of the performance evaluation index system must be objective, that is, fitting the actual situation of rural informatization construction and objectively reflecting the relationship between the various elements affecting the process of informatization construction and the role of each element. The development of rural life is a dynamic process, with new things and problems arising at every moment. To achieve a better service effect of rural informatization construction, the construction process should keep pace with the times and adjust the construction process according to the actual situation. Therefore, the performance evaluation of rural informatization construction should also be dynamic, in line with the technical level and management level of rural informatization construction, and make the most real-time and accurate evaluation of rural informatization construction:

$$
\begin{aligned}
S_{i j} & =\left\{\begin{array}{l}
S_{i j}-\left(S_{i j}+S_{\max }\right) f(t), r<0.5 \\
S_{i j}-\left(S_{i j}+S_{\min }\right) f(t), r \geq 0.5
\end{array},\right. \\
f(t) & =m(1+t) P_{\text {min }} .
\end{aligned}
$$

Moreover, from the objective function and constraints, single-objective optimization also presents linearity, so single-objective optimization is also solved using linear programming methods. Linear programming is the study of the rational arrangement of human and material resources under certain conditions so that the best economic results is an important branch of operations research earlier, faster development, and widely used, more mature methods. It is a mathematical method to assist people in scientific management, widely used in economic analysis, business management, and engineering technology, for the rational use of limited human. When one level changes, other levels respond accordingly. Sometimes the role of multiple levels needs to be coordinated by the intermediary level. It is widely used in economic analysis, business management, and engineering technology to provide a scientific basis for optimal decision-making with limited human, material, financial, and other resources. In this paper, we need to solve the potential value of each objective function, carry out the objective specification, then use the weighting method to 


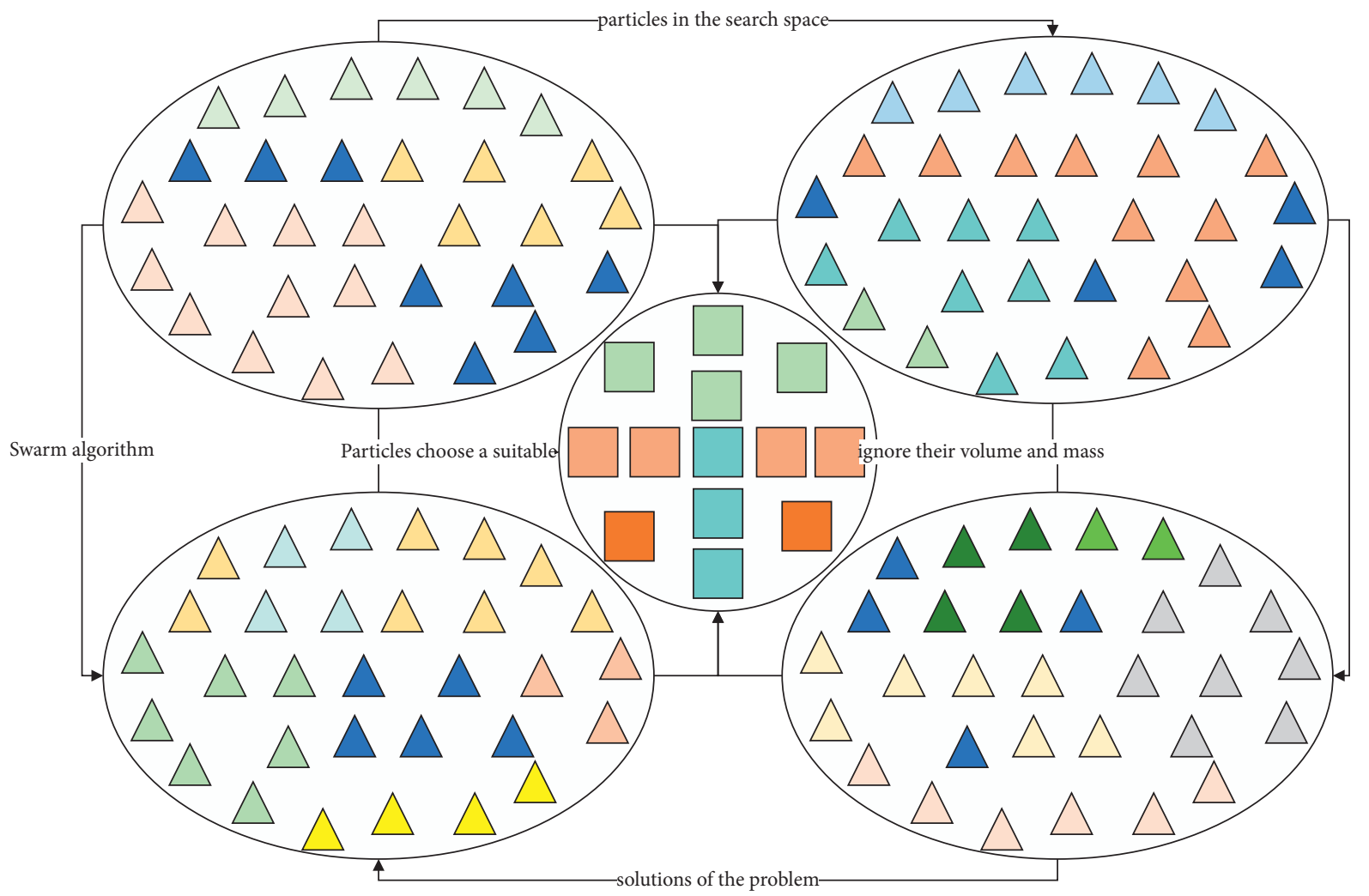

FIGURE 1: Framework of the binary fuzzy clustering analysis algorithm.

synthesize the multiobjective optimization model into a single objective function, and finally solve the linear programming of the total objective function. The objective function and constraints are relatively simple, but the intermediate solution results and the scientific and rational nature of the optimization parameters need to be observed at any time during the solution process, and the data can be adjusted according to the optimization object at any time.

The soil water resources carrying capacity index system of arable land is formulated based on comprehensive analysis and understanding of the soil water resources system, agricultural economy, agricultural ecology and environment, scientific and technological development, and regional agricultural development planning. The index system reflects the overall condition of the carried object through its overall effect, accurately grasps the soil water resources and its impact on agricultural cultivation and agricultural ecology, and provides the basic data and analytical conclusions necessary for the sustainable use of soil water resources. It provides the necessary basic data and analysis conclusions for the sustainable use of soil water resources. Therefore, it is possible to find out the key problems that exist and need to be solved in soil water resources carrying objects. The soil water resources carrying capacity indicator system for cropland can monitor the development process and changes in the indicators, reflecting the development and the effect of the implementation of relevant policies. The feedback of such information enables policymakers and decision-makers to assess the rationality and effectiveness of policies promptly, raise issues that need further research, improve or adjust policies in a targeted manner, provide a reliable method for comparing and selecting a rational utilization model of soil water resources, and provide an important basis for analysing and formulating relevant policies that is difficult to obtain in other ways:

$$
\begin{aligned}
r_{2}(t) & =X(t)+c_{1}(t), \\
\mathrm{SD} & =\operatorname{Inf}\left\{\frac{\sum h_{i j}(t)+h_{i(k+1)}\left(t^{2}\right)}{\sum h_{i j}(t)^{2}}\right\} .
\end{aligned}
$$

In comparing options, it is usually necessary to quantify, transform, and integrate various qualitative evaluation information. However, these quantification methods always fail to portray and describe the evaluation information accurately, and different quantification methods may bring different quantification results [16]. The horizontal axis represents the decision-maker number, the vertical axis represents the decision-maker's quantitative evaluation value for an option, the solid dots connected by the dotted line represent the quantitative evaluation value of each decision-maker, and the hollow dots connected by the solid line represent the quantitative value of the decision-maker group consistency evaluation information, and the traditional group decision method mainly obtains the consistency evaluation value by assigning the summation to the decision-makers, as shown in Figure 2.

The evaluation information of each decision-maker fluctuates up and down around the group consistency 


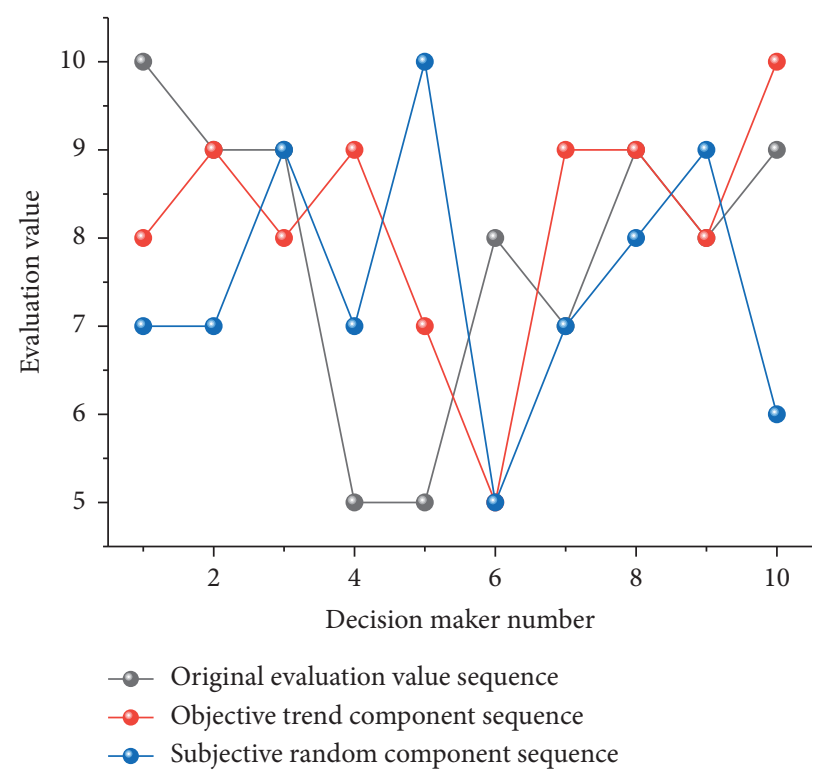

FIGURE 2: Schematic decomposition of the information sequence for group decision-making.

information, and after eliminating the variability of decision-makers, the perception of the decision group about the program tends to converge. In practice, if the decisionmakers can make full use of their perceptions to objectively evaluate the solution to be evaluated, there must be a common part of group perceptions in the evaluation information, that is, group consistency information. Group consistency information is an objective existence, which varies depending on the program to be evaluated and the evaluation criteria. It may be different from the evaluation information of any decision-maker. However, in actual decision-making, through multiple rounds of communication and other methods to gradually eliminate the cognitive differences among decision-makers, the final evaluation results can also gradually converge to the consistency information.

The objective trend component sequence in the group decision information sequence is where the consistency of group evaluation information lies and is bound to play a decisive role in the decision-making process [17]. If the subjective random components can be extracted from the group decision information sequences while retaining the objective trend components, the objectivity of the final decision results will certainly be largely improved. EMD, a data-driven adaptive nonlinear time-varying signal decomposition method with a high signal-to-noise ratio and without specifying any basis function, chosen to decompose the group decision information sequence, separate the subjective random components from it, retain the objective trend components, and prefer a decision solution based on the mean value of the objective trend component sequence as the criterion. From the basic principle of the EMD method, it is known that the process of decomposition and extraction of group decision information think sequences is data-driven and adaptive, so it can better solve the problems of subjectivity and no uniform standard of the algorithm in the traditional method of assembling group decision information and make the final decision result more in line with the objective law.

\subsection{Experimental Design for High-Performance Computing} Early Warning in Agricultural Economics. By consulting experts as an important reference basis for determining the performance evaluation indicators of rural informatization, the selection and recruitment of experts is, therefore, an important process in building the performance indicator system of rural informatization construction. To ensure the comprehensiveness and rationality of the performance index system of rural informatization construction, experts and scholars with rich experience should be selected from various fields when selecting experts. Strictly formulate the activity development process to ensure that the performance evaluation index system is fair, effective, scientific, and reasonable. This paper intends to select experts and scholars with extensive knowledge in the fields of science and technology, economics, law, and politics, as well as managers from relevant departments of county and district governments for expert consultation [18]. The selected experts will form an expert group to conduct the first round of expert group meetings to screen the previously formulated performance indicators of rural informatization construction. Each expert will give the decision results and their reasons. The expert group made general decisions on the performance evaluation indicators based on the results and reasons of each decision, improved the indicators that were not considered, and initially determined the performance evaluation indicators.

The plantation sector is extremely close to human survival and development, being the main source of food and means of subsistence for human beings, providing raw materials for the light and textile industries, the food 
industry and other industries, and fodder for animal husbandry and fisheries. It provides raw materials for the light and textile industries, the food industry, and other industries and feeds for livestock and fisheries. Information on science and technology-based agricultural services in the whole industry chain of the planting industry includes soil cultivation, selection of good seeds, sowing or seedling transplanting, seedling management, cultivation, harvesting, processing, transportation of agricultural products, and marketing, as shown in Figure 3.

Farming, which includes livestock and aquaculture, is an important source of protein nutrition. Among them, pork accounts for over $60 \%$ of meat food consumption and greatly affects people's living standards. In this paper, we summarize the information of science and technology benefiting agriculture services in the whole industrial chain of pork, including the procurement of pig feed, breeding, breeding, processing, distribution, and marketing. Based on the research on the information of science and technology benefiting agriculture services in the whole industry chain of planting and breeding, the information of science and technology benefiting agriculture services in the whole industry of agriculture is summarized [19]. The information of science and technology benefiting agriculture services in the whole agricultural industry chain includes (1) preproduction agricultural production materials, varieties, and technologies, which are provided by agriculture-related enterprises and agriculture-related science and education units; (2) inproduction agricultural production process, natural resources, and field management, which are provided by agricultural production personnel such as independent farmers and agriculture-related service units; and (3) postproduction agricultural products storage and transportation, processing and sales, which are provided by the main body providing social services, and so on. Information on sightseeing and tourism is also included. Through the analysis of the whole agricultural industry chain, it is clear that the elements involved in the information system of science and technology services for agriculture are mainly agriculture-related administrative departments, agriculturerelated science and education units, agriculture-related enterprises, agriculture-related service units, and independently operated farmers. Agriculture-related administrative departments cover the whole industry chain and provide policy support and financial funds to guide inputs. Agriculture-related science and education units provide agricultural technology innovation results. Agriculture-related service units focus on agricultural science and technology promotion services. Agriculture-related enterprises carry out agricultural business activities such as supply of agricultural materials and purchase and sale of agricultural products. Farmers operating independently produce under the guidance and support of other subjects and feedback production information, as shown in Figure 4.

Although some of the village service centres are equipped with computer facilities, they have failed to share information resources between districts because these organizations have not yet built information network platforms. Information facilities are the foundation of rural informatization construction. Among the 14 farms and towns surveyed, partly due to the backwardness of informatization infrastructure, this has led to inefficient information transfer and low-quality agricultural production and operation in these areas. In addition, the survey showed that most regions have a relatively good status of Internet equipment owned by farm households, but their role still needs to be improved [20]. The number of computers with Internet access owned by farm households amounted to $43.2 \%$, and mobile phones with Internet access amounted to $54.1 \%$. Then, the weighting method is used to synthesize the multiobjective optimization model into a single objective function, and finally, the total objective function is solved by linear programming. Some rural residents buy goods through the Internet, but the lack of construction of coordination network channels makes it very inconvenient for rural residents to pick up goods. Only a small percentage of residents have used the Internet to register for medical appointments, and the registration is only done with the help of young children or neighbours. Very few people know how to use this online information function. The main content of Internet access is for leisure and entertainment, news, facts, and chatting with friends. Very few residents use the Internet to learn about production and business information and to solve problems in their lives through online information. The Internet has a low degree of application in the lives of rural residents, with few benefits and a narrow scope. Through survey interviews with farming households, most villagers believe that the Internet is of little use in raising family income and improving living standards. Due to the imperfect information facilities and limited literacy level, the villagers' level of awareness, imitation ways, and learning channels for the application of the Internet in production and life is very limited.

\section{Results and Analysis}

4.1. Performance of Binary Fuzzy Clustering Analysis Algorithm. To verify the judgment ability of the MNNE statistic, it will be compared with the MINE statistic on several noiseless association relations in Figure 5. The results in Figure 5 show that although the two statistics have different scores on each association relation, the order of judgment on each relation of the association relation is the same. Both MNC and MIC identify the functional association relation as 1 , and the strength value of $\mathrm{MNC}$ is higher than MIC in the three compound association relations. One of the reasons is that MNC can identify the local subtle structure of the association relation. In the monotonicity deviation judgment, the higher the MAS value, the stronger the association relation is nonmonotonic. MNC_MAS has higher score than MIC_MAS on all association relations which does not strictly indicate that MNC_MAS is strict on monotonicity. Both are consistent in judging the monotonicity of the listed simple function association relations. In the monotonicity judgment of circle, the two statistics are close to 0 for their monotonicity. This is related to the symmetry of the circle. For the linear combination relationship and the composite relationship with straight lines in 


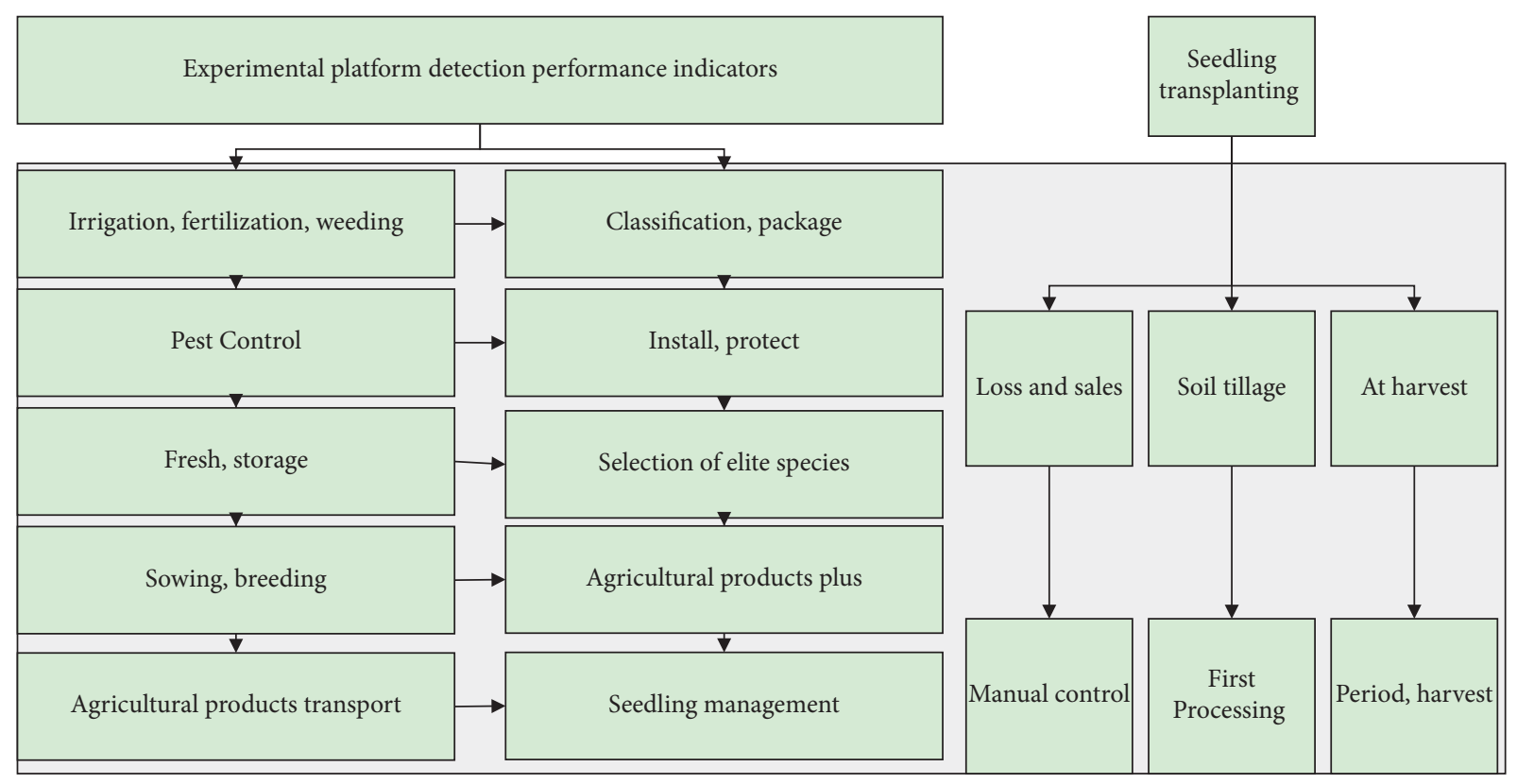

FIGURE 3: Early warning experiments for high-performance computing in the agricultural economy.
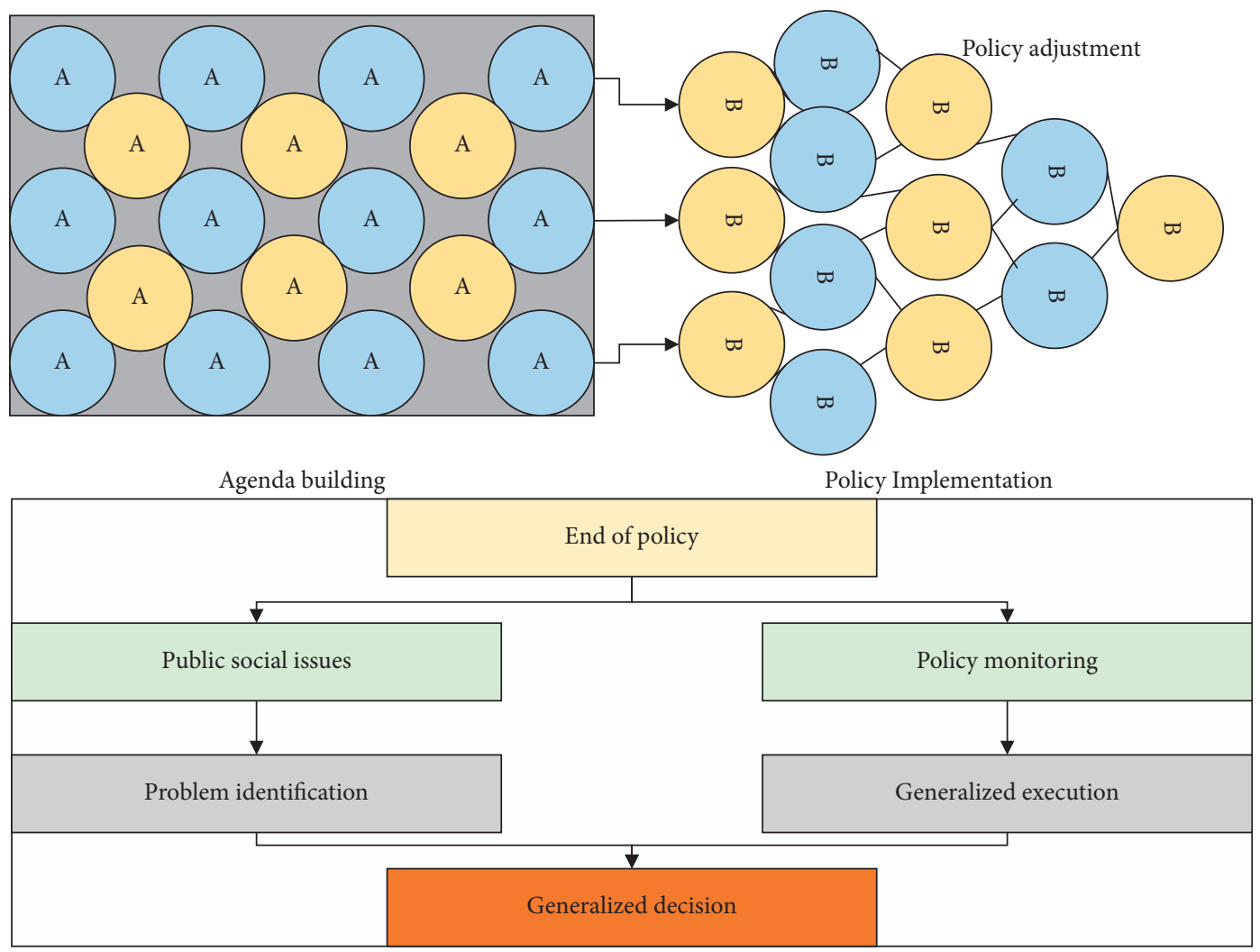

FIGURE 4: Theoretical model of the early warning process.

the circle, the two metrics have different judgments in order. The judgment of MNC_MAS considers the degree of nonmonotonicity of the composite relationship of two linear combinations is higher than that of the composite relationship with straight lines in the circle, probably the same point in the straight lines in the circle has a greater influence on the local structure. In order to determine whether the relationship between $\mathrm{MNN}$ and $\mathrm{MEV}$ is functional, we give a score of 1 on the first four common functional relationships, which indicates that the MNN can judge whether the association relationship is functional. In contrast, in the judgment of the combination function, the MNN scores 


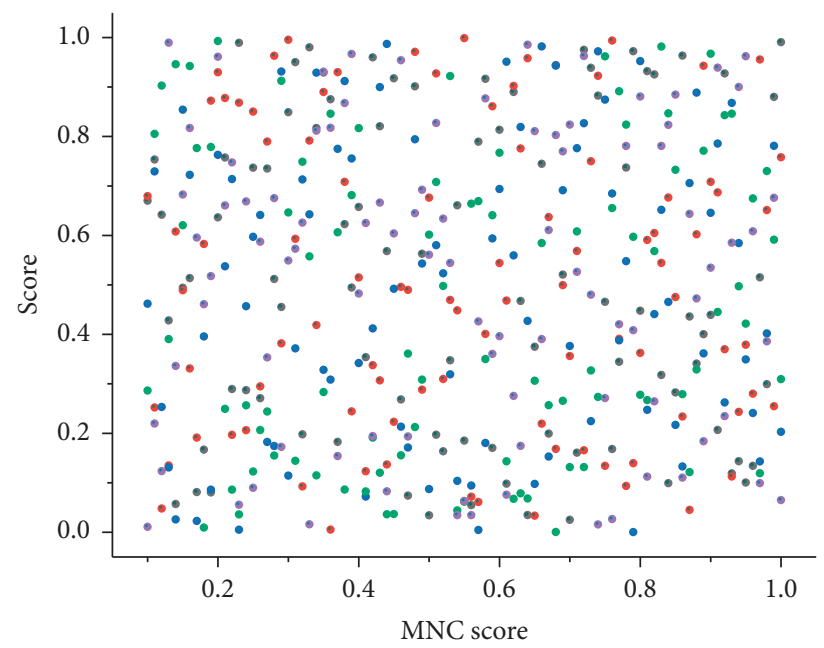

FIgURE 5: Correction of performance on the WHO dataset.

lower than the MEV indicate that the MNN is stricter in the judgment of the functional relationship. The MNB and MCN are used to capture the complexity of the association relationship, and the two judgments of the complexity of the association relationship in relative order are consistent.

However, these quantification methods are always unable to accurately describe the evaluation information, and different quantification methods may bring different quantification results. Based on the current agricultural technology, basic inputs are given to the development of the scale of water-saving agricultural technology, and the inputs should meet the development requirements of water-saving agriculture. It is expected that, by 2030, the scale of watersaving agricultural technology will be expanded to a certain extent, the crop yield and agricultural output value per unit area will be increased to a certain level, and the utilization of soil water resources will be increased to a certain level.

Based on the current agricultural technology, the development of water-saving agricultural technology scale is given greater investment efforts, and at the same time, the popularization of water-saving agricultural technology is given a corresponding increase, so that farmers pay more attention to technical water conservation, and the input can meet the requirements of good development of the watersaving agricultural scale. By 2030, the scale of water-saving agricultural technology is expected to be expanded, crop yield and agricultural output value per unit area will be increased to a larger level, and soil water resources utilization will be improved to a larger extent. Based on current agricultural technology, full investment is given to the development of water-saving agricultural technology scale, priority is given to the construction of water-saving agricultural technology-related projects in agricultural development planning, water-saving agricultural technology and popular science knowledge are vigorously promoted, and farmers are fully satisfied with the full development of watersaving agricultural scale by investing in technology to save water. It is expected that, by 2030, the scale of water-saving agricultural technology will be fully developed, as well as unit area crop yield and agricultural output value to get a great level of growth, and soil water use has been greatly improved. According to the model calculation requirements, the basic calculation parameters are tabulated and described separately, where the same data for the low, medium, and high scenario solution parameters are shown in Figure 6.

Due to the complexity of decision problems, the limited cognitive ability of decision-makers, and the uncertainty of decision-makers' cognitive expression, decision-makers' decision information is always incomplete and full of various uncertainties, and it is in such a context that uncertainty decision theory and methods are established. How to make full use of the limited decision information and effectively excavate it, construct a scientific and reasonable decision method, identify the objective value implied in the decision information to the maximum extent, and improve the efficiency and quality of decision-making is one of the key problems of uncertain decision-making research. The expert group makes a general decision on the performance evaluation indicators based on the results and reasons of each decision, completes the indicators that have not been considered, and initially determines the performance evaluation indicators. In a narrow sense, the purpose of decision-making is to select a relatively satisfactory solution from several alternative solutions to the decision problem, and the scientific basis for ranking and choosing is the quantitative value of the comprehensive evaluation of each alternative, so the comprehensive evaluation and quantitative methods have been a hot issue in the scientific research of decision-making.

When carrying out group decision-making on drought risk management with the participation of multi-interest subjects, especially when seeking opinions and suggestions from the public, the decision-makers are widely distributed, and the group of decision-makers has a large sample of random uncertainty. At the same time, due to the differences in decision-makers' knowledge structure, judgment ability, subjective preferences, and complex uncertainty of the decision problem itself, decision-makers may give different forms of preference information for the same problem, and the group decision information presents a mixture of 


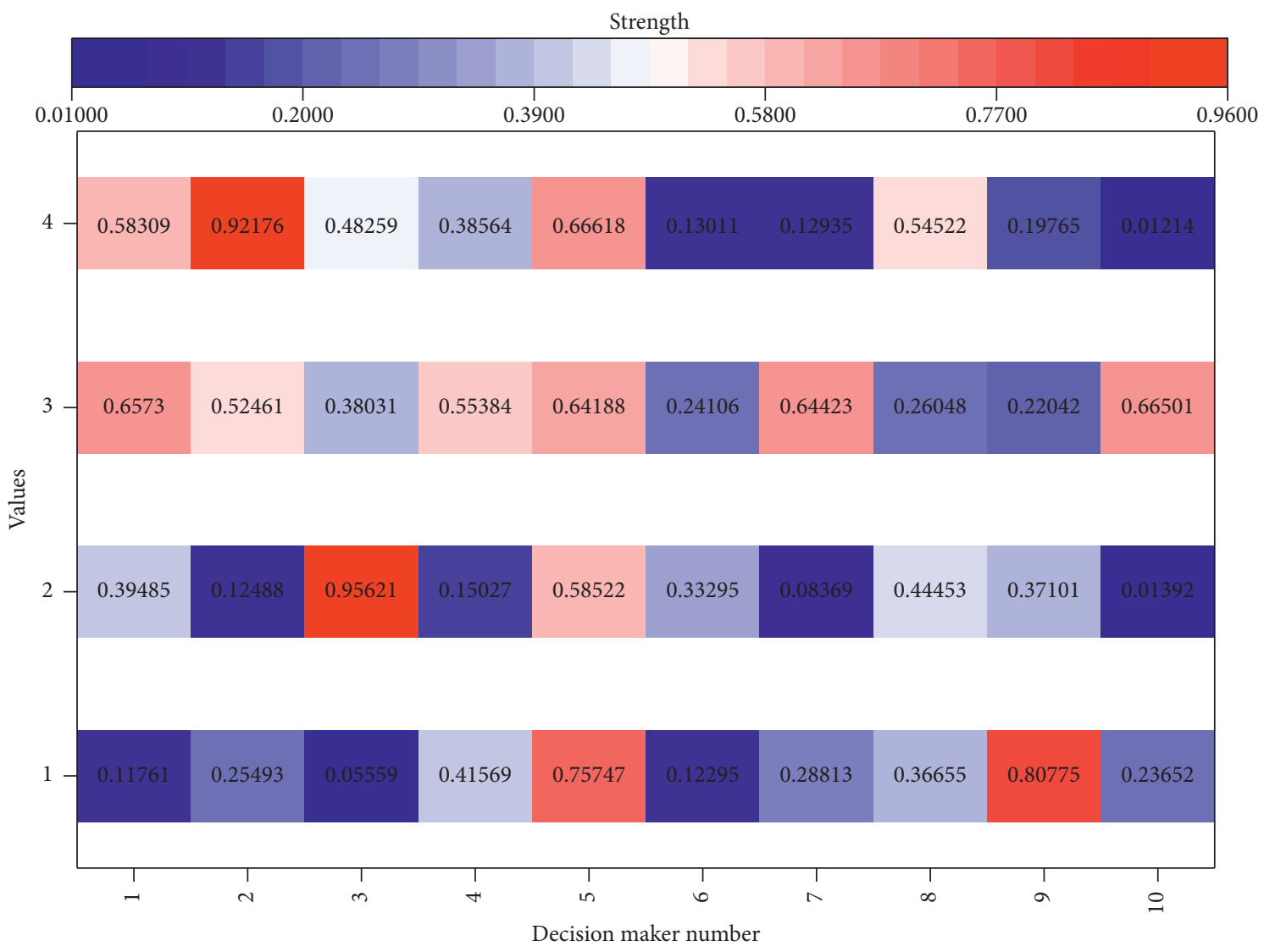

FiguRE 6: Increase in soil water use under different water-saving technologies.

uncertain information representation. If decision-makers are asked to provide decision information according to a given preference information representation (such as intuitionistic fuzzy number, and hesitation fuzzy linguistic preference relationship) and this information representation is often not the most desirable or the best for decisionmakers, or decision-makers do not understand the connotation and extension of this information representation, it may weaken the democracy and freedom of decisionmakers' judgment and even lead to distortion of decision information.

\subsection{Experimental Results of High-Performance Computing} Early Warning for the Agricultural Economy. The field observation method requires a combination of the participant and nonparticipant observation methods, mainly participant observation, to observe the natural phenomena of drought events for the proposed drought risk regulation program and study area. Investigators can select crops with relatively short growth cycles, large planting areas, and high sensitivity to drought disaster damage as field observation objects. They can observe and record crop growth and output conditions in the field throughout the growth cycle; examine farmers' farming habits, disaster response attitudes, and measures; and obtain, with the help of on-farm experiments and other nonparticipant observation methods, decision-making information.
The field questionnaire method is currently a more widely used method in social surveys at home and abroad. Compared with the field interview method and field observation method, the field questionnaire method can be designed by the questionnaire, a more comprehensive list of the proposed survey content in written form. The respondents have relatively sufficient time to think and obtain more adequate and reliable decision-making information. The questionnaire method can break through the constraints of time and space, in a wide range, the survey of many respondents at the same time. More importantly, because the questionnaire is designed following the expected format and statistical scale of the investigator, the decision information obtained is more convenient for quantitative research. The engineering measures mainly include increasing the construction of water-saving renovation and supporting projects in irrigation areas, promoting sloping land renovation projects, and promoting the application of high efficiency.

The water-saving irrigation technology and vigorous development of facility agriculture are shown in Figure 7.

The fuzzy identification model was used for comprehensive water quality evaluation, water eutrophication evaluation, and organic pollutant evaluation using water quality evaluation criteria. It was found that the eigenvalue of the mouth of the Unkey River inlet during the flat-water period using the fuzzy clustering iterative model was 2.071 with an evaluation grade of slight pollution. In contrast, the eigenvalue using the fuzzy identification model was 1.998 


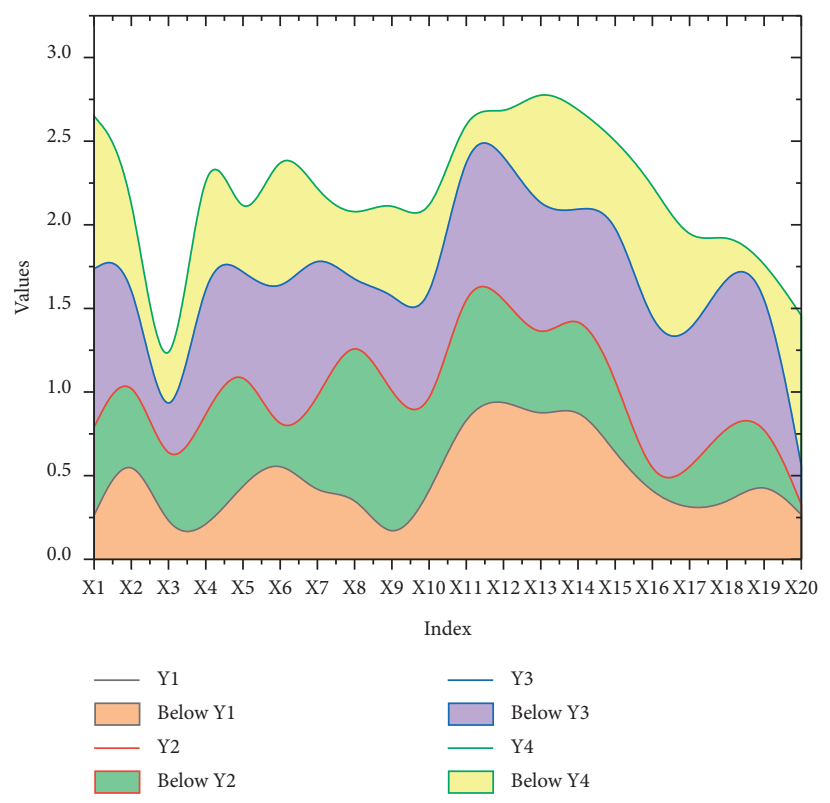

FIGURE 7: Optimal fuzzy clustering centre of water quality indicators for each period in Tausan Reservoir with the presence of inverse order.

with an evaluation grade of fair clean. The evaluation level is light pollution, while the characteristic value when using the fuzzy recognition model is 1.998 , and the evaluation level is still clean. The evaluation grades of other periods and monitoring points are consistent with the results of the fuzzy clustering iterative model and fuzzy clustering recognition model, respectively. The evaluation grades for the remaining periods and each monitoring site were consistent with the results of the fuzzy clustering iterative model and the fuzzy clustering identification model, respectively. Although the evaluation grades of the Makana River inlet are not consistent, the difference in their eigenvalues is very small and acceptable. Meanwhile, when the fuzzy identification model was used to evaluate the eutrophication of water bodies in Tausan Reservoir, the eigenvalue of 1 in the reservoir at the monitoring site during the dry period using the fuzzy clustering iterative model was 3.050, with an evaluation grade of moderate eutrophication. However, the eigenvalue of 2.971 using the fuzzy identification model had an evaluation grade of mild eutrophication, and the evaluation grades of the remaining periods and monitoring sites were consistent. In the evaluation of organic pollutants in the water body of Tausan Reservoir using the fuzzy identification model, the eigenvalue of the fuzzy clustering iterative model for the municipal intake at the monitoring site in the dry period is 1.999 , with an evaluation grade of average, while the eigenvalue of the fuzzy identification model is 2.012 , with an evaluation grade of slight pollution. The evaluation grades of the rest of the periods and the monitoring sites are the same. The reason for the difference is the same as the reason for inconsistency in the comprehensive evaluation of water quality. In summary, the optimal fuzzy clustering centre obtained by using the fuzzy clustering iterative model solution as the water quality evaluation index

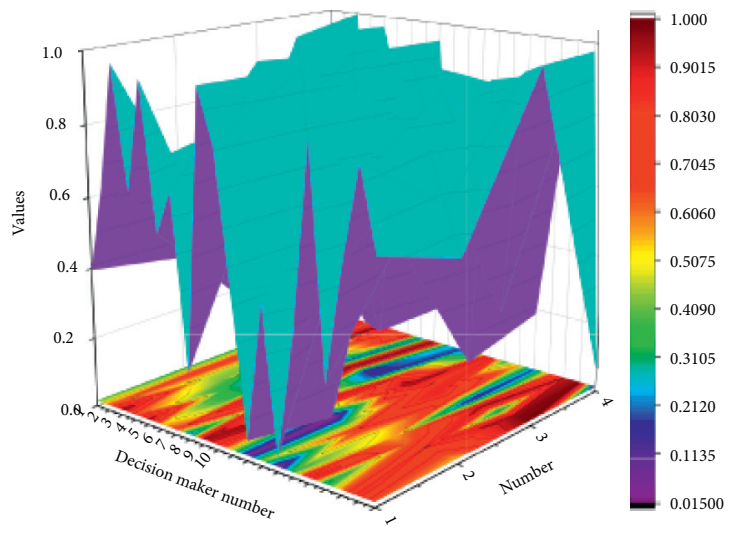

FIGURE 8: Early warning evaluation expectations by region.

for water quality evaluation is accurate and highly feasible, as shown in Figure 8.

However, the penetration rate of household broadband installation, home computers, Internet computers, and other tools to speed up informatization is not high and is still at the starting level. In addition, the information service sites in the region are sound and can provide agricultural information services such as agricultural consultation, weather forecasts, and disaster warnings to farmers. Eleven farms, four farms, and seven farms have achieved outstanding results in the development of special industries using information technology, realizing the rapid development of special industries with planting as the core. In these areas, the infrastructure arrangement is sound, and the use of the Internet has enabled the rapid transmission of information between farms and farms, integrated the existing resources to maximize resource utilization, expanded sales channels using rural e-commerce platforms, and gradually formed their industrial chains. 


\section{Conclusion}

By employing experts in different fields, group meetings and group decision-making are conducted, and experts make suggestions on the structure of the performance indicator system. This study refers to the experts' suggestions, while based on the idea of layering the construction of performance index system (target layer, element layer, specific system layer), through the grasp of the performance evaluation index system as a whole, with the basic framework of tree-like index system structure, the construction of the performance evaluation index system of rural informatization construction is completed, which can reflect the current situation of rural informatization construction in a more comprehensive way. At the same time, experts rate the importance of the finalized evaluation indexes, calculate the importance of each index, and determine the weight of each index according to the experts' opinions, which makes the evaluation results more accurate. This paper introduces interval type-two fuzzy numbers to process the data. As an extension of traditional fuzzy numbers, interval type-II fuzzy numbers inherit the characteristics of traditional fuzzy numbers to describe uncertainty and describe a higher degree of uncertainty information than traditional fuzzy numbers, which makes the results more accurate. Therefore, this study constructs an evaluation model of rural informatization construction performance based on interval type-two fuzzy numbers, by referring to the interval typetwo fuzzy number aggregation operator for all index values, and finally obtains the evaluation value of each region and ranks it by calculating its expectation. The larger the expectation value is, the more satisfied the farmers and rural enterprises in the region are with the local rural informatization construction. The smaller the expectation value is, the more the rural informatization construction in the region still needs to be improved. An empirical study is conducted to verify the effectiveness of the evaluation method proposed in this paper and its application value. The study was conducted based on the method proposed in the paper, and conclusions were drawn. The empirical study verifies the validity of the evaluation method proposed in this paper and makes suggestions for improving the performance of rural informatization construction based on the study's findings.

\section{Data Availability}

The data used to support the findings of this study are available from the corresponding author upon request.

\section{Conflicts of Interest}

The author declares no known competing financial interests or personal relationships that could influence the work reported in this paper.

\section{References}

[1] H. Ait Issad, R. Aoudjit, and J. J. P. C. Rodrigues, “A comprehensive review of data mining techniques in smart agriculture," Engineering in Agriculture, Environment and Food, vol. 12, no. 4, pp. 511-525, 2019.

[2] C. Si and Z. Xiaomei, "Optimization of regional forestry industrial structure and economic benefit based on deviation share and multi-level fuzzy comprehensive evaluation," Journal of Intelligent and Fuzzy Systems, vol. 37, no. 1, pp. 145-157, 2019.

[3] S. Sankaranarayanan, M. Prabhakar, S. Satish, P. Jain, A. Ramprasad, and A. Krishnan, "Flood prediction based on weather parameters using deep learning," Journal of Water and Climate Change, vol. 11, no. 4, pp. 1766-1783, 2020.

[4] J. Yang, C. Wang, and B. Jiang, "Visual perception enabled industry intelligence: state of the art, challenges and prospects," IEEE Transactions on Industrial Informatics, vol. 17, no. 3, pp. 2204-2219, 2020.

[5] M. I. Bhatti and H. Q. Do, "Recent development in copula and its applications to the energy, forestry and environmental sciences," International Journal of Hydrogen Energy, vol. 44, no. 36, pp. 19453-19473, 2019.

[6] M. K. Tripathi and D. D. Maktedar, "A role of computer vision in fruits and vegetables among various horticulture products of agriculture fields: a survey," Information Processing in Agriculture, vol. 7, no. 2, pp. 183-203, 2020.

[7] S. Bhatore, L. Mohan, and Y. R. Reddy, "Machine learning techniques for credit risk evaluation: a systematic literature review," Journal of Banking and Financial Technology, vol. 4, no. 1, pp. 111-138, 2020.

[8] Q. Yue, F. Liu, C. Song, J. Liang, Y. Liu, and G. Cao, "Exploration and application of the value of big data based on data-driven techniques for the hydraulic internet of things," International Journal of Embedded Systems, vol. 12, no. 1, pp. 106-115, 2020.

[9] L. Li, P. Jiang, H. Xu, G. Lin, D. Guo, and H. Wu, "Water quality prediction based on recurrent neural network and improved evidence theory: a case study of Qiantang river, China," Environmental Science and Pollution Research, vol. 26, no. 19, pp. 19879-19896, 2019.

[10] H. Abedi Gheshlaghi, B. Feizizadeh, and T. Blaschke, "GISbased forest fire risk mapping using the analytical network process and fuzzy logic," Journal of Environmental Planning and Management, vol. 63, no. 3, pp. 481-499, 2020.

[11] W. A. Ali, K. N. Manasa, M. Aljunid, M. Bendechache, and P. Sandhya, "Review of current machine learning approaches for anomaly detection in network traffic," Journal of Telecommunications and the Digital Economy, vol. 8, no. 4, pp. 64-95, 2020.

[12] B. G. Buchanan and D. Wright, "The impact of machine learning on UK financial services," Oxford Review of Economic Policy, vol. 37, no. 3, pp. 537-563, 2021.

[13] X. Jiang, N. Zhang, J. Huang, P. Zhang, and H. Liu, “Analysis of prediction algorithm for forest land spatial evolution trend in rural planning," Cluster Computing, vol. 24, no. 1, pp. 195-203, 2021.

[14] W.-H. Su, "Advanced machine learning in point spectroscopy, RGB- and hyperspectral-imaging for automatic discriminations of crops and weeds: a review," Smart Cities, vol. 3, no. 3, pp. 767-792, 2020.

[15] X. Ji and J. Lu, "Forecasting riverine total nitrogen loads using wavelet analysis and support vector regression combination model in an agricultural watershed," Environmental Science and Pollution Research, vol. 25, no. 26, pp. 26405-26422, 2018.

[16] S. Sun, C. Wang, H. Ding, and Q. Zou, "Machine learning and its applications in plant molecular studies," Briefings in functional genomics, vol. 19, no. 1, pp. 40-48, 2020. 
[17] M. Zhao, D. Li, and Y. Long, "Forestry big data platform by Knowledge Graph," Journal of Forestry Research, vol. 32, no. 3, pp. 1305-1314, 2021.

[18] B. N. Subudhi, D. K. Rout, and A. Ghosh, "Big data analytics for video surveillance," Multimedia Tools and Applications, vol. 78, no. 18, pp. 26129-26162, 2019.

[19] M. Arif, K. K. Alghamdi, and S. A. Sahel, "Role of machine learning algorithms in forest fire management: a literature review," Journal on Robotics and Automation, vol. 5, no. 1, pp. 212-226, 2021.

[20] Z. Chen, L. D. Van Khoa, E. N. Teoh, A. Nazir, E. K. Karuppiah, and K. S. Lam, "Machine learning techniques for anti-money laundering (AML) solutions in suspicious transaction detection: a review," Knowledge and Information Systems, vol. 57, no. 2, pp. 245-285, 2018. 